\title{
Note sur les helminthoses des animaux domestiques reconnues à Madagascar
}

\author{
par P. DAYNES \\ Laboratoire de Tananarive-Madagascar
}

\begin{abstract}
RÉSUMÉ
L'auteur présente une revue des Helminihes des animaux domestiques de Madagascar.

Ceux-cl sont présentés par espèce domeshıue avec leur fréquence et leur imporiance. Quelques Helrnınthoses secondaires sont également cıtées.
\end{abstract}

\section{AVANT-PROPOS}

Nous tenons à rappeler que ce relevé des Helminthes connus chez les animaux domestiques à Madagascar est surtout un travail de compilation.

Nous avons puisé abondamment dans les rapports annuels du Laboratoire.

Certaines diagnoses d'espèces faites autrefols ne nous ont pas toujours paru très convaincantes bien que nous les ayions signalées.

II serait très utile de reprendre beaucoup de déterminatıons et de les faire revoir par des spéciatistes à la lumière des données récentes de la Systématique.

Nous pourrions alors constituer une collection valable des différents spécimens d'Heiminthes de Madagascar.

\section{INTRODUCTION}

Madagascar est située de part et d'autre du Tropique du Capricorne, entre les $12^{\mathrm{e}}$ et $26 \mathrm{e}$ parallèles Sud, et à cheval sur le $45^{\mathrm{e}}$ degré de Longitude Est. $6.600 \mathrm{~km}$ de côtes entourent une île de
$1.600 \mathrm{~km}$ de long et $500 \mathrm{~km}$ de large, couvrant $590.000 \mathrm{~km}^{2}$.

Madagascar, située donc pratıquement en zone intertropicale, présente du fait de sa géographie des différences climatiques importantes. Celles-ci se traduisent par la localisation de certaines infestations en foyers lımıtés.

En simplifiant au maximnm, on peut délmiter 3 grandes régions géographiques ef climatiques: D'abord la Côte Est, étroite et très humide ( $3 \mathrm{~m}$ de pluie à Tamatave); ensuite les Plateaux dont le relief d'origine volcanique est souvent tourmenté. L'altitude (de 800 à $1.500 \mathrm{~m}$ ) y engendre un climat plus tempéré. Enfin, la région Ouest descendant lentement des Plateaux, beaucoup plus sédimentaire et moins tourmentée: cette région chaude, humide au Nord et au Centre, devient plus sèche et subdésertıque vers le Sud ( $400 \mathrm{~mm}$ de pluie à Tuléar).

Rappelons que l'isolement de l'île lui fait posséder une faune souvent particulière.

Par le terme d'Helminthose nous entendons ici aussi bien l'infestation que la maladie. 
Nous nous proposons de présenter les prıncipales Helminthoses des animaux domestiques avec leur fréquence ef leur importance. Nous citerons également, pour ces mêmes espèces domestıques, les Helminthes d'importance secondaire reconnus d̀ Madagascar.

\section{1. - HELMINTHOSES DES BOVINS}

Le cheptel bovin compte à Madagascar 6 à 9 millions de têtes $(6,3 \mathrm{M}$ recensement adminıstratif 1962 et $9 \mathrm{M}$ estimation mission LACROUTS 1961). Ces bovins qui représentent une grande partie de la richesse de quelques 5 millians d'habitants sont presqu'exclusivement des zébus. Il existe autour des grandes villes, Tananarive plus particulièrement, un noyau de métissage avec des races laltières européennes.

Les jeunes anımaux sont ceux qui paient le plus lourd tribut aux Helminthoses. Les adultes sont moins parasités et surtout moins affectés par leur parasitisme. La mortalıté des jeunes, parfois très élevée, peut être attribuée en grande partie d̀ l'action des parasites qui affaiblissent, souvent consıdérablement, l'animal quand ils ne sont pas la cause directe de la mort. Quelles sont donc les Helminthoses rencontrées chez les bovins à Madagascar ?

\section{Ascaridose}

L'Ascaridose par Neo-ascaris vitulorum (GOEZE, 1782) atteint surtout les jeunes anımaux des élevages semi-ıntensifs ou intensifs où l'infestation peut se produire au pâturage. Dans les élevages extensifs (de loin les plus nombreux) l'infestation semble se produire au parc, les animaux parqués étant plus souvent infestés que les animaux laisssés en liberté la nuit. Le « par cà bœufs » est d'ailleurs un excellent milieu pour l'œuf d'Ascaris (sol meuble, aéré, légèrement humide).

Les coproscopies effectuées dans un élevage semi-intensif montrent que 35 p. 100 des veaux sont porteurs d'Ascaris. Dans le même troupeau le taux d'animaux adultes infestés par l'Ascaris est inférieur à 5 p. 100. Les infestations sont parfois importantes. Un veau de $28 \mathrm{~kg}$, à la mamelle, était porteur de 41 Ascaris d'un poids total de $80 \mathrm{~g}$.
Différents sondages en élevage extensif traditionnel donnent des taux d'infestation souvent inférieurs à 1 p. 100 chez les adultes et parfois aussi faibles chez les jeunes. Chez les anımaux métis laitiers des environs de Tananarive, l'Ascaridose semble également peu fréquente, même chez les jeunes. Une enquête aussi sévère et serrée que possible est actuellement en cours afin d'obtenir des chiffres exploitables.

Si les accidents tragiques avec mortalité (perforation, occlusion) ne sont pas monnale courante, par contre la baisse de l'état général est fréquente. Les jeunes animaux présentent des troubles intestinaux avec diarrhée, profitent mal, ont une croissance nettement retardée.

Le traitement que l'on conseille est le drogage systématıque à l'Adipate de piperazine, utılisé à la dose de $30 \mathrm{cg}$ par $\mathrm{kg}$ de poids vif et répété 3 fois.

Ce traitement appliqué sur les animaux âgés de : 1 mois, 2 mols et 3 mols, nous donne de bons résultats.

\section{Hémonchose}

Ce parasitısme de la caillette, dû à Hoemonchus contortus (RUDOLPHI, 1803) est parfois fréquent et intense dans certaines régıons côtières de l'île.

Les jeunes animaux de l'année, atteints par cefte affection, peuvent être porteurs de milliers de parasites dans la caillette, alors qu'on n'en trouve que quelques rares exemplaires chez les adultes infestés.

Les symptômes sont ceux d'une anémie avec diarrhée pouvant conduire à la cachexie et à la mort.

Le traitement à la Phenothiazine donne de bons résultats de même que le traitement au Tetrachlorethylène (BUCK et GRÉTILLAT, 1957) (6).

\section{Bunostomose}

Le parasite en cause est Bunostomum phiebotomum (RAILLIET, 1900). D'une façon générale, ce parasite plutôt rarement rencontré se trouve un peu partout dans l'île de Madagascar. Cet Ankylostomidé s'est cependant montré très pathogène sur un troupeau de veaux dans la région du Lac Alaotra, où il semble plus fréquent qu'ailleurs. 
Le Tetrachlorethylène a donné des résultats intéressants dans ce cas particulier (POISSON et BUCK, 1937) (29).

\section{Autres « strongyloses» (S. L.) gastro-intestinales}

- Esophogostomum radiatum (RUDOLPHI, 1803) est fréquemment rencontré dans tout Madagascar. II est rarement très pathogène.

- Esophagostomum columbianum (CURTICE, 1890 ) a été signalé chez les bovins (BUCK et GRETILLAT, 1957) (6).

- Cooperio pectinato (RAMSON, 1907) est rencontré assez souvent mais, semble-t-il, sans provoquer de symptômes (mêmes auteurs).

- Trichostrongylus sp. est signalé chez les bovins (Rapport Labelva 61).

\section{Autres Nématodes du tube digestif des bovins}

- Strongyloïdes papillosus (WEDL, 1856) est fréquemment rencontré. II se trouve parfois en assez grand nombre dans l'ıntestın grêle des bovins mais Il s'agit le plus souvent de Strongyloïdose-infestation et non de Strongyloìdosemaladie, Les cas d'infestation massive de jeunes bovins par S. papillosus, se traduisent par une diarrhée nauséabonde, un amaigrissement et un retard de croissance. La Phenothiazine ne semble pas agir. La Methyridine nous a donné quelques bons résultats (observations non publiées).

- Trichuris sp. (T. discolor.) est signalé chez les bovins de Madagascar (Rapports Labelva).

\section{Dictyocaulose}

Cette Metastrongylose est due à Dictyocaulus viviporus (BLOCK, 1782). Sur les Plateaux (BUCK et GRÉTILLAT, 1957) (6) signale la fable intensité des infestations expliquant l'absence de symptômes alors que SOUPRE en 1948 signalait sur ia Côte Ouest (Majunga) une épizootie grave mais localisée.

\section{Filarioses}

- Onchocerca gutturasa (L. G. NEUMANN, 1910) a été rencontré très fréquemment au début du siècle dans le Nord de l'île (CHRÉTIEN, 1920) (12). (1921) (13). Sa localisation est le tissu conjonc- tif entourant le ligament cervical et les ligaments articulaires carpiens ou tarsiens. CetOnchocerque n'est pas pathogène.

- Setaria labiato-papillosa (ALEXANDRINI, 1838 ) est très souvent rencontré sur les bovins de Madagascar dans la cavité péritonéale. Elle semble vivre en commensale avec son hôte (GRÉTILLAT, 1957) (19). II faut noter cependant qu'une infestation importante (100 sétaires) et des localisations erratiques ont été rendues responsables d'accidents (KRICK, 1930 cité par POISSON et BUCK, 1936) (28).

\section{Spiruroses}

- Thelazia sp. a été signalé dès 1923 (TIS$S \mid E ́$, 1924) (31) comme responsable d'Ophtalmies chez les bovins à Madagascar. Il ne, semble pas cependant que ce parasite soit très fréquent.

\section{Paramphistomoïdoses}

- Poramphistomum cervi (SCHRANK, 1790) reconnu depuis longtemps à Madagascar, (GRETILLAT, 1959) (23) se rencontre fréquemment chez les bovins (GRÉTILLAT, 1958) (22) et parfois leur rumen en contient $u$ n très grand nombre sans que l'on décèle pour autant des troubles même légers.

- Poramphistomum bothriophoron' (STILES et GOLDBERGER, 1910) (bien étudié par GRÉTILLAT en 1957) (20) se rencontre moins souvent et en moins grand nombre que $P$. cervi. Son rôle pathogène semble également restreint.

- Cormyerius spatiosus (BRANDES, 1898) et Carmyerius dollfusi (GOLVAN CHABAUD et GRÉTILLAT, 1957) (18) sont hématophages. Aussi leur rôle pathogène ne peut-il être négligé dans les infestations importantes comme l'a montré GRÉTILLAT en 1957 (21) ; ils seraient rarement rencontrés sur les Plateaux.

\section{Autres Trématodes}

- Eurythrema pancreaticum (JANSON, 1889) est connu à Madagascar. Son importance est secondaıre. II sévit sur la Côte Est surtout et seuls les jeunes bovins très parasités, présentent quelques symptômes de diarrhée et d'amaıgrıssement (BUCK et FLORENCE, 1938-1939) (5) (FLORENCE, 1939) (15). 


\section{Monieziose}

Due d̀ Moniezia exponsa (RUDOLPHI, 1810), cette Helminthose est peu à peu reconnue dans les différentes régions de l'île : Sud-Ouest et Sud au climat assez sec, Lac Alaotra au climat plus humide et moins chaud, Moyen-Ovest et Ouest de l'île (Miarinarivo et Tsiroanomandidy d'une part, Majunga d'autre part).

La Monieziose semble présenter une recrudescence en fin de saison des pluies, époque vers laquelle la plupart des jeunes bovins atteignent 4 à 6 mois. Le plus souvent, ce sont les animaux de 4 à 12 mais qui sont porteurs de Moniezia dans leur intestin grêle. En général, ce parasitisme est assez bien supporté même lors d'infestation relativement importante ( $290 \mathrm{~g}$ de Moniezio chez un veau de 5 mols, en très bon état). Parfois cependant, cet Helminthe se montre pathogène et peut entraîner un amaigrissement et un retard de croissance très sensible.

Dans les régions où la Monieziose existe depuis peu, il semble que les pâturages s'infestent de plus en plus ; les pourcentages d'infestation seraient en progression d'année en année, dès que l'on a reconnu Moniezıa dans une zone déterminée.

Le problème soulevé ici nécessite que des sondages importants et réguliers soient effectués pendant plusieurs années.

\section{Cestodoses larvaires}

La ladrerie bovine serait rarement rencontrée à Madagascar. Elle a été cependant signalée de temps en temps ( 8 fois entre 1910 et 1963).

- Cysticercus bovis (forme larvaire du Tenia saginata) a été identifié en 1910, 1933, 1956 et 1959. Cette rareté explique peut-être que l'on n'alt pas encore identifié Tenia saginata comme agent du Téniasıs chez les Malgaches.

- L'Echinococcose-hydatidose est par contre moins rare. On la rencontre chez les bovins dans toutes les régions de l'île. Pour le seul abattoir de Tananarive en 1959, les saisies, par le Service d'Inspection Sanitaire, pour Echinococcose chez les bovins onł porté sur 0,6 p. 100 des poumons et 0,2 p. 100 des foies. L'Echınococcose affecte peu l'animal et a surtout une incidence économıque. N'oublions pas cependant son inci- dence sur la santé de l'homme (BUCK et COURDURIER, 1962) (7).

- Cysticercus tenuicollis a été vu en 1930 sur le diaphragme du bœuf (Rapport Labelva).

\section{Helminthose aberrante}

- En 1931, un Gigantorhynchus sp. aurait été constaté dans la cavité péritonéale d'un bovin, accroché à la séreuse de l'intestin (Rapport Labelva, 1931).

\section{II. - HELMINTHOSES DES PORCINS}

L'élevage du porc à Madagascar est un élevage traditionnellement important. Il a connu de grandes vicissitudes avec l'explosion de la Maladie de Teschen. Celle-ci est maintenant «contrôlée» grâce à la vaccination. L'élevage porcin s'améliore chaque jour et accroît régulièrement son importance économique. On estime le cheptel moyen actuel entre 450.000 et 500.000 porcs.

On élève le porc surtout sur les plateaux, dans les provinces de Tananarive ef de Fianarantsoa, ainsi qu'au Lac Alaotra. Les zones de cultures semi-intensıves ou intensives, sans avoir l'exclusivité de l'élevage porcin, sont celles où l'élevage «amélioré » connaît un essor encourageant.

Le porc est amélıoré par métissage avec des races européennes précoces d'une part, et par perfectionnement des modes d'élevage d'autre part.

Le métissage est avancé dans les secteurs où la vulgarisation des méthodes modernes, a pénétré. Dans ces mêmes secteurs, les modes d'élevage évoluent et l'on abandonne peu à peis l'élevage ancestral pour un élevage mieux conduit.

Dans ces élevages, à allure industrielle (rarement) ou à caractère familial, les animaux sont parqués et nourris, souvent avec une alimentation qui cherche à être rationnelle. Le petit éleveur sait maintenant ce qu'est une provende, aussi bien que le propriétaire d'une importante porcherie.

Par contre, l'élevage ancestral qui est le plus important, est toujours du type «porc coureur » « agent voyer du village ».

On se trouve donc devant deux variétés de l'élevage du porc et la parasitologie $y$ est parfois différente. Mals dans les deux cas, le porc est 
fréquemment l'objet d'une des Heiminthoses que nous allons étudier.

\section{Ascaridose}

Cefte infestation, due à Ascaris suum (GOEZE, 1782) nous semble mériter la tête de liste, tant elle est répandue et parfois intense. Les jeunes porcelets de race locale, coureurs, sont infestés d'Ascaris, dans 60 p. 100 des cas. Le pourcentage d'infestation tombe par contre très bas dans certains élevagese de type industriel où les animaux sont déparasités assez régulièrement.

En 1963, 15 p. 100 des coproscopies effectuées au Service d'Helminthologie du Laboratoire, montrent des œufs d'Ascaris, et 20 p. 100 des animaux parasités le sont par l'Ascaris. Ces coproscopies ont porté aussi bien sur des animaux de brousse non améliorés, que sur des animaux d'élevage en expérimentation dans les Services de Nutrition ou de Virologie et déparasités.

Dans la région de Miarinarivo, de petits élevages familiaux, à peine améliorés (métissage, animaux sur la terre, provende mal équilıbrée), ont une Ascaridose qui atteint 15 à 20 p. 100 des animaux. On arrive mème parfois à constater jusqu'à 25 p. cent d'animaux parasités en élevage surveillé. Les pourcentages précités, proviennent des coproscopies.

L'intensité de l'infestation est variable. Elle est rarement très faible. Cing à dıx Ascaris sont souvent rencontrés chez les porcelets infestés. Quarante à cinquante Ascaris ne sont pas chose rare chez des porcelets de 4 à $5 \mathrm{~kg}$. Parfois, cette infestation massive permet de dénombrer une centaine d'Ascaris. L'obstruction intestınale est plus fréquente que la perforation. La congestion intestinale intense provoquée par la présence des Ascaris, dans l'intestın grêle en général, peut alier jusqu'à l'hémorragie intestinale. On connaît également l'obstruction du canal cholédoque par un ver, obstruction qui conduit à l'ictère, la plupart du temps, cependant les conséquences de l'Ascaridose ne sont pas brusquement tragiques. Le rôle pathogène de l'Ascaris est plus insidieux, s'installant discrètement. Il ne conduit pas à la mort des individus, mais compromet, très sensiblement, la rentabilité de l'élevage. Mauvais état général, amaigrissement, retard de croissance devenant impossible à rattraper, sont le lot des animaux infestés.

Les traitements que nous pratiquons sont le drogage à l'Adipate de piperazine $(25 \mathrm{cg} / \mathrm{kg})$ et l'administration de Tetrachlorethylène $(0,20$ à $0,25 \mathrm{~cm}^{3} / \mathrm{kg}$ ). Nos essais (en cours de publication) confirment l'activité de l'Hygromycine B. D'autres observations (non publiées) effectuées avec la méthyridine ne nous ont pas donné des résultats très intéressant's.

It convient de rappeler ici que l'infestation de l'homme par l'Ascaris (A. lumbricoides) atteınt un pourcentage aussi élevé que chez le porc (au moins 60 p. 100). La majorité de la population malgache est rurale et vit dans des conditions d'hygiène toutes relatives qui la font vivre souvent près des porcs. Il est donc utile que soit résolu, de façon irréfutable et définitive, le problème de l'unıcité ou de la dualité d'Ascaris lumbricoïdes.

\section{Macracanthorhynchose}

L'Echinorhynque parasite du porc est Macracanthorhynchus hirudinaceus (PALLAS, 1781). Les coproscopies effectuées au Laboratoire, en 1962 et 1963, donnent respectivement 5 p. 100 et 2 p. 100 de porcs infestés par Macracanthorhynques, mais ces chiffres doivent être, interprétés. Nous avons vu, à propos de l'Ascaris, que nos coproscopies avalent porté sur des porcins de toutes sartes. La Macracanthorhynchose est en fait plus souvent rencontrée que ces chiffres ne l'indiquent. Les coproscopies effectuées sur des animaux de brousse peu améliorés, donnent 7 p. 100 d'infestation. Les autopsies effectuées sur des porcelets de race locale, type coureurs, nous montrent que l'infestation esł plus fréquente. Sur sept porcelets autopsiés dernièrement, quatre, dont deux de façon importante, étaient infestés de Macracanthorhynques, alors que la coproscopie n'avait rıen décelé de cette Helmınthose. Chez ces quatre porcelets âgés de 3 à 6 mois, les Échinorhynques étaient, précısons le, de jeunes exemplaires dont les plus gros ne dépassaient pas 8 à $9 \mathrm{~cm}$.

II nous semble que les porcs supportent assez bien l'infestation par Acanthocéphales. Des anımaux autopsiés, pour diverses raisons, au Service des diagnostics, portaient dans leur intestin un certain nombre de Macracanthorhynchus, déjà 
nettement visibles de l'extérieur par leurs nodules de fixation, sans que l'état général soit excessivement altéré. La perforation de l'intestin par ces parasites est rencontrée et provoque une péritonite mortelle.

Nous ne connaissons pas de traitement efficace. La prophylaxie doit lutter contre la larve de hanneton, hôte intermédiaire ; elle est possible dans les élevages surveillés et entretenus propres.

\section{Esophagostomose}

Elle se rencontre avec une certaine fréquence. Elle est due à (Esophagostomum dentatum(RUDOL$\mathrm{PHI}, 1803$ ) et 'Esophogostomum longicaudum (GOSDEY, 1925). Ces Helminthes ne semblent pas se montrer très pathogènes pour le porcelet ou le porc. La coproscopie permet de trouver l'cesophagostomose imaginale. Lorsqu'on désıre la confirmer par l'autopsie, on découvre quelques œsophagostomes (5 à 10) et quelques nodules œsophagostomiens sur la paroi colique.

L'autopsie de porcs permet quelquefois de rencontrer l'œsophagostomose à son stade le plus pathogène. La paroi du colon montre de nombreux nodules larvaires, œsophagostomiens, mais, à moins d'infestation massive, les animaux ne paraissent pas en avaır extrêmement souffert.

L'on sait que le traitement de l' $\mathcal{F}_{\text {sophagosto- }}$ mose à son stade pathogène est difficile, l'élément infestant étant «protégé » (et le diagnostic de certitude n'existant pas). Le traitement (prophylactique donc) de l'(Frophagostomose au stade imaginal des parasites, utilise le Tetrachlorure de carbone ef la Phénothıazıne. Nous avons essayé l'Hygromycine B et confirmé son efficacité sur les Esophagostomes alors que l'Adipate de piperazine ne nous a pas paru très efficace.

Dans les infestations très importantes, I'CEsophagostomose peut donc gêner le développement harmonieux du porc mais son rôle néfaste se trouve surtout être du domarne économique. Les «boyaux» à nodules deviennent inutılısables en charcuterie.

\section{Uncinariose}

Cette Helminthose mérite une mentıon spéciale. Elle n'est pas courante chez le parc. Cependant quelques foyers ont été reconnus : sur les plateaux 3 foyers dons la région d'Antsirabé et un foyer au B. D.P. A. ; entre les plateaux et la côte Est, à Périnet. Uncinaria stenocephalo (RAlLLIET, 1884) se trouve parfois en grand nombre sur la muqueuse de l'Intestin grêle ef il est à peine besoin de rappeler qu'alors son action pathogène anémiante est considérable.

Un Necator surllus (-Necotor omericanus) signalé sur un porc à Antsirabé en 1931 (Rapport Labelva), est peut-être à placer dans le cadre de cette Uncinariose.

\section{Autres nématodoses gastro intestinales}

- Assez fréquemment rencontrée est la Trichurose (ou Trichocephalose) due à Trichuris trichura (LINNE, 1771) ou 'peut-être à T. suss (si ces deux espèces ne peuvent être ramenées à une seule) (EUZÉBY, 1961) (14).

Les infestations sont parfois importantes. Cette Helminthose paraît cependant bien supportée.

Les Anthelminthıques, habituellement utilisés, n'agissent guère, sauf peut-être l'Hygromycine B.

- Plus fréquemment rencontrée est la Strongyloidose due à Strongyloïdes ransomi. Elle peut atteindre plus de 80 p. 100 des animaux dans des élevages en liberté. Elle se traduit beaucoup plus rarement par des symptômes de diarrhée. Un piqueté hémorragique de l'intestin, assez serré dans les infestations massives, prouve qu'elle n'est pas à dédaigner complètement.

Le Thiabendazole que nous n'avons pu essayer serait le seul Anthelminthique pratiquement utilisable contre cet Helminthe.

- La Trichostrongylose de l'estomac, due à Hyostrongylus rubidus (HASSAL et STILES, 1892) est connue à Madagascor (Rapports Labelva).

- Signalons enfin les spiruroses gastriques du porc, dues à Physocephalus sexalatus (MOLIN, 1860) et à Arduenna strongylina (RUDOLPHI, 1819) dont les rôles pathogènes sont peu importants (GEOFFROY ef POISSON, 1930) (16).

\section{Métastrongylose}

La Strongylase pulmonare du porc est le fait à Madagascar de Metastrongylus salmi (GOEDELST, 1923), Choerostrongylus pudendotectus (WOSTOKOV, 1905) et Metastrongylus madagoscariensis (CHABAUD et GRÉTILLAT, 1956) (11).

Les infestations sont fréquentes et parfois massives chez les porcelets élevés sur les parcours humıdes (ver de terre, hôte intermédiaire). 
Elles sont (logiquement) bien morns fréquentes dans les porcheries cimentées. Les jeunes sont plus sensibles que les adultes. Quelques traitements actifs sont connus. Le traitement pratique facilement utilisable (intervention unique, si possible collectıve) est encore à trouver. La prophylaxie est facile. Le diagnostic, à notre avis, doit toujours êrre confirmé par la coproscopie. Beaucoup d'affections pulmonaires sont trop souvent rapportées aux Metastrongles.

\section{Stéphanurose}

Cette affection est due à Stephonurus dentotus (DIESING, 1839) signalé en 1916 à Madagascar par CAROUGEAU (10). On la rencontre sur la Côte Est avec fréquence, dans le Sud, sur les plateaux (Antsirabé-Lac Alcotra), dans le Nord et dans l'Ouest de l'île. On peut donc considérer qu'elle est partout présente à Madagascar.

Le parasite se situe habituellement dans le tissu périrénal où il peut entraîner la formation d'abcès et de trajets purulents. L'animal supporte assez bien ce parasitisme, sauf dons quelques rares cas d'infestations massives qui entraînent dépérissement et mort.

Le diagnostic peut être fait par analyse microscopique des urines. La prophylaxie réside dans la propreté des élevages.

Du point de vue économique, ce sont les saisies d'ordre sanitaire qui sont à considérer. Plus de 50 p. 100 des reins des porcs, en provenance du Lac Alaotra, sont saisis à l'abattoir de Tananarive. La graisse périrénale est en principe saisie en même temps. Une localisation plus rare du stéphanure dans le foie entraîne parfois la saisıe de celui-ci. La saisie totale pour viande urineuse est très rare.

\section{Cestodoses larvaires}

Cysticercose, Hydatidose, Sparganose sont les cestodoses larvaires du porc.

- La Cysticercose est due à Cysticercus cellulosoe (forme larvaire de Tenia solium). La ladrerie peut atteindre jusqu'à 24 p. 100 des porcs selon les régions ( 8 à 24 p. 100 d'après les statistiques de l'Inspection sanitaire en 1958). Les saisies totales pour ladrerie chez le porc étaient de 2,58 p. 100 des porcs abattus à l'abattoir de Tananarive en 1960.
- L'Hydatidose-Echinococcose est due à Echinococcus polymorphus, forme larvaire de Echinococcus gronulosus. Elle est nettement moins fréquente chez le porc que chez le bouf.

- La Sparganose serait due à la forme larvaire du Bothriocephale du chien. La présence de Spargonum est connue depuis longtemps chez le porc où il se localise au niveau du dıaphragme surtout et partout ailleurs (GEOFFROY et POISSON, 1932) (17).

Rares sont les études faites à Madagascar sur la Sparganose (et la Bothriocephalose correspondante). BUCK d'une part (Rapports Labelva, 1935-36), CAPRON et BRYGOO en 1960 (8) d'autre part, sont les seuls à notre connaissance à avoir étudié ce problème.

\section{III. - HELMINTHOSES DES OVINS ET DES CAPRINS}

Les élevages ovin et caprin sont localisés surtout au Sud et au Sud Ouest de l'île et remontent sur la côte Ovest où une population musulmane (Comoriens) en entretient en permanence.

Les statistiques du Service de l'Elevage estimaient en 1962 les ovins à 230.000 et les caprins à 250.000. Ces chiffres sont très variables d'une année d̀ l'autre (recensement plus ou moins rigoureux, mortalité par disette très variable, abattage ef consommation en fonction des récolfes).

Le métissage a été tenté dans le Sud (Ambovombe ef Beloha) avec le bélier mérinos sur les ovins autochtones (grosse queue) pour la production de viande et dans le Sud Ouest (AmpanihyOuest) avec des boucs angora sur la chèvre locale, pour la production de mohair. Sur la Côte Ouest, l'élevage reste celui d'animaux tout venant pour la consommation courante.

Plusieurs Helminthoses sont connues chez ces animaux. Quelques-unes se sont montrées parfois graves ou même meurtrières. Ce sont l'Hemonchose et la Monieziose.

\section{Hémonchose}

Due à Hœmonchus contortus (RUDOLPHI, 1803), cette strongylose de la caillette sévit surtout sur les agneaux. Anémie et entérite sont le lot de cette parasitose et conduisent à la mort dans les infestations massives où la caillette est tapissée 
de parasites. On trouve très fréquemment des Infestations plus ou moins légères. On a diagnostiqué l'Hémonchose sur les ovins du Sud et sur ceux du Lac Alaotra.

\section{Monieziose}

Elle est le faif de Moniezıa exponsa (RUDOL$\mathrm{PHI}, 1810$ ) et s'est montrée pendant quelques années, particulièrement grave sur les chevrecux de la région d'Ampanihy en se traduisant par une mortalité élevée et, chez les adultes métissés, une baisse de la production de mohair (BUCK ef GRÉTILLAT, 1957) (6). Ce parasitisme semble actuellement en nette régression sauf peut-être dans la région Sud entre Ampanihy et Beloha. En 1959 \& 1960, nous n'avons pas trouvé une seule Moniezia sur les autopsies des caprins du Sud-Ouest.

\section{Esophagostomose}

Cette infestation mérite une mention, non du fait de sa gravité mais de sa fréquence chez les Ovins. Elle est due à $\mathcal{C E s}_{\text {sophagostomum }}$ colombianum (CURTICE, 1890) surtout, et plus rarement à 0 . venulosum (RUDOLPHI, 1809). On connaît également à Madagascar l'Esophagostomose caprine signalée sur le chevreau en 1954 (Rapport Labelva).

\section{Autres Nématodoses}

- Nous signalerons d'abord la Strongyloïdose, Strongyloidose-infestation et non Strongyloidosemaladie, due à Strongyloïdes (papillosus).

Nous l'avons rencontrée dans la région de Tananarive, tant sur les ovins que sur les caprıns.

- L'infestation par Nematodirus filicollis (RUDOLPHI, 1802) est signalée dès $1926 \mathrm{chez}$ la chèvre et en 1930 chez le mouton (Rapport Labelva).

Nous avons rencontré une Nematodirose sur des caprins importés d'Afrique du Sud dans le Sud et le Sud Ouest de l'île. Pour cette infestation reconnue grâce d̀ une Coproscopie caractéristique, il ne nous a pas été possible de savoir quelle est l'espèce en cause, n'ayant pu obtentr d'Helminthes.

-De 1958 à 1960, ont été reconnus Trichostrongyius sp., Bunostomum trigonocephalum et Cooperia sp. (Rapports Labelva).
- L'infestation par Trichures est connue depuis longtemps.

\section{Trématodoses}

- L'infestation par Paramphistomum cervi chez les ovins est déjà citée dans les rapports du Service de l'Elevage de 1930 . Elle a toujours été reconnue comme assez fréquente dans certaines régions et pratiquement non pathogène.

- Le rapport annuel 1931 du Laboratorre de l'Elevage signale chez le mouton Gastrothylax crumenifer et en 1957 BUCK et GRÉTILLAT sıgnalent la Gastrothylose ovine à Carmyerius (6)

- Citons pour mémoire les distomatoses à Fasciola hepatica (LINNE, 1758) et à Dicrocoelium lanceolatum (RUDOLPHI, 1803) toujours rappelées, mais qui n'ont été rencontrées que sur les ovins importés de France. Ces parasitoses ne se sont pas étendues, très probablement par absence des hôtes intermédiaires indispensables.

\section{Cestodoses larvaires}

- Cysticercus tenuicollis, forme larvaire du Tenia hydatigena du chien, est rencontré dans les séreuses des Ovins et des Caprins.

- Echinococcus polymorphus, forme larvare d'Echinococcus granulosus, est rencontré dans divers organes des Ovins ef en particulier dans le foie.

Ces Cestodoses larvaires ont un rôle économique du fait des saisies qu'elles peuvent entrấner à l'abattoir.

- Citons une Cysticercose péritonécle et testiculaire, observée chez un ovin en 1938 (Rapport Labelva).

\section{Autres Helminthes parfois signalés}

(Rapports et publications technıques Labelva). Nous nous bornerons à les citer :

- Ascaris lumbricoides.

- Chabertia ovina.

- Trichostrongylus axel et $T$. colubriformis.

- Ostertagio circumancto et $O$. ostertagi.

- Protostrongylus rufescens.

- Mullerius capillaris.

- Dictyocaulus filaria.

- Metastrongyius elongatus (exceptionnel chez le mouton). 


\section{IV. - HELMINTHOSES DES ÉQUIDÉS}

L'élevage des équidés, chevaux, ânes et mulets, est relativement restreint d̀ Madagascar.

Les chevaux élevés surtout sur les plateaux (Tananarive ef Fianarantsoa) sont des chevaux de selle, assez petıts ( 3 d̀ $400 \mathrm{~kg}$ ). Quelques-uns finissent leur carrière en tirant, dans la banlieue de Tananarive, des voiturettes de transport en commun. Rares sont ceux dont la chair est consommée.

Les ânes, malgré leurs qualités indéniables, n'ont pas réussi à s'implanter. Ils étendent leur aire de distribution plus vers le Sud.

Quelques mulets, comme c'était à prévoir, ont vu le jour.

On compie environ 1.800 chevaux et 200 ânes pour tout Madagascar.

Différentes Helmınthoses ont cependant attaqué cet élevage peu important en nombre.

\section{Strongyloses intestinales}

Elles sont dues aux Strongles, sensu stricto, ainsi qu'à des Triodontophorus.

Strongylus vulgaris (LOOS, 1900) et S. equinus (MULLER, 1780) sont reconnus depuis longtemps (Rapports Labelva).

Strongylus edentotus (LOOS, 1900), Triodontophorus serratus (LOOS, 1900) et $T$. brevicauda (BOULENGER, 1916) ont été signalés en 1957 par BUCK et GRETILLAT (6). Ces Strongles intestinaux sont fréquents chez le cheval et entraînent parfois un mauvais état général de leur hôte. Nous traıtons soit à la phénothiazine à faibles doses répétées, soit avec un mélange de phénothiazine-adipate de piperazıne. Nous conseillons surtout les traitements périodiques, les animaux étant toujours gardés en milieu de réinfestation possible (et même probable). Nous ne sommes pas parvenus à faire une distinction valable entre les œufs des Strongylus et de Triodontophorus lors des coproscopies.

\section{Trichonemose}

La Trichonemose, signalée par BUCK en 1935 (3), associée en général aux strongyloses citées ci-dessus, est fréquente. Elle serait due en particulier d̀ Trichonema tetraconthum (LOOS, 1900) cité par POISSON en 1927 (25). Si elle est fréquente, elle ne semble cependant pas très pathogène.

Un autre Trichoneminé, Gyalocepholus capifotus, a été reconnu en 1959 (Rapport Labelva).

En 1962, le Dr DIAZ UNGRIA a identifié :

Cyathostoma catinatum (LOOS, 1900).

C. minutum (YORKE et MACFIE, 1918).

C. nassotum (LOOS, 1900).

C. insigne (BOULANGER, 1917).

C. longibursatum, (YORKE et MACFIE, 1918).

C. colicatum, (LOSS, 1900).

\section{Spirurose gastrique}

Cette Helminthose est due à la présence dans l'estomac du cheval de 3 habronèmes, Habronema megastoma, $H$. muscae et $H$. microstama, tous 3 cités à Madagascar (Rapports Labelva).

Le 1er de ces spiruridés provoque des tumeurs du cul de sac droit de l'estomac, mais ne semble pas affecter davantage les animaux que les 2 autres habronéminés qui eux, sont libres dans l'estomac.

On a signalé des plaıes d'été dues à l'Habronemose larvaire.

\section{Dictyocaulose}

Dictyocoulus arnfieldi (COBBOLD, 1884) est signalé chez le cheval en 1934 (Rapport Labelva). Rarement rencontré, ce métastrongle pourrait être à l'orıgine d'affections pulmonaires.

\section{Autres Nématodoses}

- BUCK, dans un relevé d'Helminthes effectué en 1934, signale qu'une Setariose due à Setario hemorragica a été observée par POISSON dans la région de DIEGO-SUAREZ en 1931.

- Nous avons rencontré la Strongyloidose, due à Strongyloides sp. (westeri ?) sur des animaux de la région de Tamatave.

- L'Oxyurose, due à Oxyuris equi (SCHRANK, 1788) se rencontre assez souvent. Parfols, son intensité est suffisante pour provoquer un mauvais état général de l'animal. Nous traitons avec l'Adipate de piperazine, administré à la sonde naso-œsophagienne. Ce traitement, complété par une série de lavements à base d'adipate de piperazine, est renouvelé quelques semaines plus tard. 
- L'Ascaridose, rencontrée surtout chez les jeunes, mais parfois aussi chez l'adulte, est due à Parascaris equorum (GOEZE, 1782). Elle peut être à l'origine de coliques. Nous traitons à l'Adipate de piperazıne.

\section{Gastrodiscose}

Cette trematodose due à Gastrodiscus aegyptiacus (COBBOLD, 1876) est relativement fréquente. Le diagnostic coproscopique est facile. Le traitement ne s'impose pas souvent tant les animaux semblent garder leur bon état d'entretien. En 1911 CAROUGEAU (9) en trouvant le parasite d̀ l'autopsie de 2 mulets a pu noter que l'un de ces deux animaux était porteur de milliers de gastrodisques fixés sur le colon sans avoir présenté de symptômes.

$\mathrm{Si}$ le traitement est nécessaire (amaigrissement), nous utilisons le Verbutane administré à la sonde-naso-œsophagienne.

\section{Cestodose}

En 1928 GEOFFROY signale un Anoplocephalo perfoliata dans le coecum d'un cheval à Tananarive (Rapport Labelva, 1930).

\section{V. - HELMINTHOSES DES CARNIVORES DOMESTIQUES}

Les chiens ef chats sont à Madagascar des familiers de l'homme, surtout le chien. Bequcoup d'Helminthoses sont connues chez ces animaux. Elles ont été étudiées surtout sur les animaux des villes soit qu'ils aient été amenés par leurs propriétaires à la Clinique proche du Laboratoire soit que, errants, ils aient été attrapés par le Service Municipal et mis en fourrière.

\section{Ascaridose}

Cette affection est très fréquemment rencontrée. 20 p. 100 des chiens de Tananarive montrent des cufs d'Ascaris dans leurs excréments. Ont été reconnues comme espèces parasites en cause, Toxocara canis (WERNER, 1782) et Toxascoris leonina (LINSTOW, 1902); le premier semble plus fréquent que le second. Les animaux, jeunes souvent, mais ce n'est pas une règle, sont ordinairement très affectés par cette Helminthose qui est la pluparł du temps associée à l'Ankylostomose.

Chez le chat les infestations par' Ascaris sont plus rares. Les Toxocara cati (SCHRANK, 1788) sont parfois rencontrés en grand nombre chez les chatons en très mauvais état.

Nous trattons à l'Adipate de piperazine ou au Tetrachlorethylène.

\section{Ankylostomose}

L'infestation du chien par Ankylostome est plus fréquente que la précédente. On note plus de 30 p. 100 des coproscopies positives sur les chiens de Tananarive. La fréquence de l'Ankylostomose est encore plus élevée sur la Côte Est.

Ce parasitisme est en général associé à l'Ascaridose. Il est dû à Ankylostoma coninum et à Uncinario stenocephola. II éprouve parfois beaucoup les animaux. Nous traitons au Tetrachloréthylène.

Notons qu'Uncinaria stenocephala est signalé chez le porc (BUCK et GRÉTILLAT, 1957) (6) et que l'Ankylostome est probablement responsable de beaucoup de dermites humaines observées sur la Côte Est.

\section{Trichurose}

La Trichurose diagnostiquée par la coproscopie atteint près de 20 p. 100 des chiens. Elle semble très peu pathogène (Infestation pure).

Elle est due à Trichuris vulpis.

\section{Spiruroses}

- La Spirocercose canine est due d̀ Spirocerco sanguinolenta (RUDOLPHI, 1819). Elle est connue depuis 1921 à Madagascar (POISSON, 1927) (25).

Elle est relativement fréquente. Les coproscoples effectuées en 1963 l'ont montrée dans 5 p. 100 des cas. Elle est, d'autre fois soupçonnée à l'examen clinique ou découverte à l'autopsie. On peut donc conclure qu'il y a nettement plus de 5 p. 100 des chiens infestés de Spirocerques.

La symptomatologie, très variable, est très classique. Les localisations aortiques et gastriques sont connues. Nous sommes pratiquement démunis du point de vue thérapeutique.

- La Spirurose gastrique du chat, due à Spirura ritypleurites (DESLONGCHAMPS, 1824), 
a été signalée également chez un chaton de Tananarive en 1958 (Rapport Labelva).

\section{Strongyloïdose}

L'existence d'une Strongyloidose canine due à un Strongyloides sp. différent de S. stercoralis est à l'étude. Les cas d'infestation ne semblent pas fréquents. La Strongyloïdose par Strongylordes stercorolis est signalée chez le chien en 1956 et en 1961 (Rapport Labelva).

\section{Filariose}

La Dirofilariose du chien, due à Dirofilarıa immitis (LEYDY, 1856) est connue depuis longtemps à Madagascar (Rapport Labelva, 1930). Elle est fréquente en région côtière chaude et humide.

A côté de la mıcrofilaire de D. Immitis on trouve souvent dans le sang du chien de ces mêmes régions une autre microfilaire. Un projet de recherche est à l'étude afin de déterminer la filaire en cause.

\section{Angiostrongylose}

Des larves ont été rapportées à Angiostrongylus en 1962 (non publié).

\section{Cestodoses}

Le chien est fréquemment parasité par les Cestodes. D'après nos autopsies au moins 20 p. 100 des chiens de Tananarive sont porteurs de Cestodes. 15 p. 100 des coproscopies effectuées sur le chien nous montrent à Tananarive la présence du Bothriocéphale.

- La Bothriocéphalose qui est donc fréquente a été longtemps attribuée à Diphyllobothrium latum (LINNÉ 1758) puis à D. Latum, D. erınacei et D. mansonoïdes. Il semble établi actuellement qu'elle est due, en partie tout au moins, à D. erinacer europaer (RUDOLPHI, 1819) (BAVAY, 1890) (1), (JOYEUX, BAER et GAUD, 1950) (24), (BRYGOO et CAPRON, 1960) (2).

$\|$ y a là un problème qu mérite d'être repris sous l'angle «cycle» et sous l'angle statistique par une enquête de masse. Le traitement que nous essayons est l'administration de Dichlorophène.
La Bothriocéphalose du chat, sıgnalée en 1936 (Rapport Labelva) a été rapportée en 1952 à D. erinacer europaei et en 1959 à D. Latum (Rapports Labelva, 1952 et 1959).

- Dipylidium canınum (LINNÉ, 1758) est assez fréquemment rencontré chez le chien. Nous avons pu constater des infestations massives chez les chiens de fourrière à Tananarive. Nous essayons dans ce cas également le traitement par le Dichlorophène.

D. coninum est également cité chez le chat (Rapports Labelva).

- Sont également signalés (Rapports Labelva) Tenia hydatigena (PALLAS, 1766).

Tenia pisiformıs (BLOCH, 1780).

Mesocestoides lineatus (GOEZE, 1782).

Echinococcus gronulosus (BATSCH, 1786)

ainsi que Tenia teniaeformis (BATSCH, 1786) (chat) et Mesocestoïdes litteratus (BATSCH, 1786) (chatte) (collection).

- Notons une cestodose larvaire : en 1930 Cysticercus cellulosae fut trouvé dan's le muscle cardiaque d'un chien (Rapport Labelva).

\section{VI. — HELMINTHOSES DES VOLAILLES}

L'élevage des poules et poulets est très développé. Il s'agit bien souvent d'oiseaux vivant autour des habitations dans les villages de campagne. Rarement ces animaux sont bien nourris. Ils picorent çà ef là.

Autour des grandes villes et plus particulièrement autour de Tananarive, on trouve des élevages en parquets, en batteries, bien conduits ef destinés à fournir œufs ou viande. De nombreuses Helminthoses se rencontrent dans l'un et l'autre cas.

Nous citerons les principales infestations parasitaires rencontrées chez les poulets ainsi que les espèces mises en cause. Le cas échéant nous citerons les Helmınthes parasitant les oiseaux de basse-cour autres que les gallinacés.

\section{Hétérakidoses}

Elles sont fréquentes chez les poules ef l'infestafion est le plus souvent intense ou même massive.

Grâce à la coproscopie, on la rencontre déjà chez plus de 30 p. 100 des volailles. 
Elles sont dues à Ascaridia galli (SCHRANK, 1788) et à Heterakis gallinarum (SCHRANK, 1788). Ascaridia colombae est signalé chez le pigeon (Rapport Labelva, 1930).

\section{Syngamose}

Cette infestation reconnue depuis longtemps (POISSON, 1927) (25) a fait autrefols de gros ravages dans les élevages de poules. Syngamus trachea est le parasite en cause. S'il semble provoquer actuellement moins de dégâts qu'autrefois, il est cependant loin d'être rarement rencontré.

\section{Aufres Nématodoses}

Sont signalées les infestations par :

- Capillaria sp. (Rapports Labelva).

- Capillaria retusum (?) (Rapports Labelva).

- C. gallina (Rapports Labelva).

- Amidostomum anseris (Oie) en 1933 (Rapports Labelva).

- Amidostomum sp. (Ole) en 1961-62 (Rapports Labelva).

- Acuario spiralis (POISSON, 1931) (26).

- Tetrameres fissispina (BUCK, 1939) (4).

- Oxyspiruro sp. (BUCK et GRÉTILLAT, 1957) (6).

- Oxyspirura mansonı (PIntade) (Rapport Labelva, 1957).

\section{Cestodoses}

Egalement fréquentes, elles se présentent souvent sous forme d'infestations intenses.

Les Cestodes reconnus sont :

- Davanea proglottina.

- Roilliettina penetrans.

- R. tetragono.

- R. cesticillus.

- R. volzi.

- R. echinobothrido.

- Choanotenia sp. ?

- Amoebotenia sphenoides (POISSON, 1934) (27).

- Hymenolepis.
- H. serrata (Pigeon).

- H. meleagris (Dindon).

- Cotugnia daynesi (QUENTIN, 1963) (30).

- Octopetolum sp.

- O. gutturae (Pintade).

- Aporino delafondi (Tourterelle).

- Tetrathyridium variabile, forme larvaire de Mesocestoïdes, dans le pouman et dans le muscle d'une poule.

\section{Trématodoses}

Sont signalées les infestations par:

- Prosthogonimus (Rapport Labelva, 1948).

- Echinostoma revolutum (Poule, Pintade. Canard, Dinde).

- Tracheophilus cymbius (Canard) (Rapport Labelva, 1962).

- Philophtolmus gralli (Publication technique Labelva).

Il est peut-être bon de noter que les Helminthes des oiseaux domestiques sont certainement loin d'être tous connus, que les espèces en cause doivent être identifiées avec rigueur et que les hôtes intermédıaires le cas échéant, sont en général inconnus.

\section{VII. - HELMINTHOSES DES LAPINS}

L'élevage des lapins est très restreint à Madagascar. Tout au plus trouve-t-on quelques clapiers de petite envergure auprès des grandes villes.

Les Helminthoses reconnues sont très rares.

- La plus anciennement connue et la plus fréquente est l'infestation par Cysticercus pisiformis forme larvaire du Tenia pisiformis du chien.

- En 1956, GRÉTILLAT observe une infestation massive à Possolurus ambiguus (RUDOLPHI, 1819).

- En 1958, est signalé Trichostrongylus retortaeformis sur le rapport annuel du Laboratoire.

- En 1963, une coproscopie a montré des œufs de type «Trichostrongylidés ».

\section{SUMMARY}

A note the helminthosis of domestic animals knower in Madagascar

The author presents a review of Helminths of domestic animals of Madagascar. Here they are presented with domestic species and their occurence and importance. Some secondary helmınth infections are also mentioned. 


\section{RESUMEN}

Nota sobre las helmintosis de los animales domésticos reconocidas en Madagascar

El autor pasa en revista los helmintos de los animales domésticos de Madagascar. Se presenta cada especie doméslica con su frecuencia y su importancia. Se cila también algunas helmintosis secundarıas.

\section{BIBLIOGRAPHIE}

1. BAVAY (M.). - Sur la présence du Bothriocephalus latus à Madagascar. Bull. Soc. Zool. de France, $1890,15,134-135$; in Joyeux et Coll. 1950.

2. BRYGOO (E. R.) et CAPRON (A.). - Faune parasitalre helminthologique malgache ; aperçu liminaire - Miscellanea Helminthologica Madagascariensis. Arch. 'Inst, Past. Madagascar, 1960, 28, 181-188.

3. BUCK (G.). - Note sur la Cylicostomose maladie parasitaire des équidés existant à Madagascar. Bull. econom. de Madagascar, 1935, p. 196.

4. BUCK (G.). - Un parasite des poules nouveau pour Madagascar, Tetrameres fissispina. Bull. Soc. Path., exot., 1939, 32, p. 447.

5. BUCK (G.) et FLORENCE. - Note sur un parasite du pancréas des bovins nouveau pour Madagascar. Soc. Scl. Méd. Madagascar, Séance du 29 novembre 1938 et Gozette méd. de Modogascar, 1939, 6, p. 27.

6. BUCK (G.) et GRETILLAT (5.). - Les helminthes pathogènes des animaux domestiques à Madagascar. C. R. 3e Congrès P. I. O.S. A., 1957, Section B, Tananarive.

7. BUCK (G.) et COURDURIER (J.). - Les zoonoses à Madagascar. Rev. Elev. Méd. Vet. Poys trop., 1962, 15, 181-191.

8. CAPRON (A.) et BRYGOO (E. R.). - Sparganose expérimentale de Lemur fulvus. Miscellanea Helminthologica Madagascariensis. Arch. Inst. Past. Madagascar, 1960, 28, 189195.

9. CAROUGEAU. - Sur l'existence de Gastrodisque de Sonsine à Madagascar. Bull. Soc. Sci. Méd. Madogascar, 1911, p. 9.

10. CAROUGEAU. - Présentation du Stephanurus dentatus. Bull. Soc. Scl. Méd. Madagascor, 1916, p. 31.
11. CHABAUD (A, G.) et GRETILLAT (S.). Metastrongylus madagascariensis $n$. sp. Quatrième espèce de strongle pulmonaire chez le porc domestique. Ann. Parasit, hum. Comp., 1956, 31, 572-577.

12. CHRÉTIEN (A.). - L'onchocercose du bœuf à Madagascar. Bull. Soc. Cent. Méd. Vet. et Rec. Med. Vet., 1920, 96-168.

13. CHRETIEN (A.). - Onchocercose du Zébu de Madagascar et du bouf de France. Rev. Path. Comp., 1921, 21-46.

14. EUZEBY (J.). - Les Maladies Vermineuses des Animaux domestiques. TI, Nemathelminthes, fasc. 1er, p. 33. Vigot éd. 1961.

15. FLORENCE (R.). - Existence chez les bovins de Madagascar de l'Eurythrema pancreaticum. Bull. Soc. Path. exot., 1939, 32, 446-447.

16. GEOFFROY et POISSON (H.). - Un nouveau cas de Physocephalose de l'estomac du Porc. Bull. Soc. Sci. Med. Madagascar in Bull. Soc. Path. exot., 1930, 23, 874-875.

17. GEOFFROY et POISSON (H.), - Sparganose du porc à Madagascar. Rec. Méd. Vet. exot., 1932, 5, 21-22.

18. GOLVAN (Y.), CHABAUD (A. G.) et GRETILLAT (S.). - Carmyerius dollfusi n. sp. (Trematoda, Gastrothylacidae) parasile des bovidés à Madagascar. Ann. Poras. hum. Comp., 1957, 32, 56-70.

19. GRETILLAT (S.). - Quelques filarioses des animaux domestiques à Madagascar. Comm. 3 Congrès P. I. O S. A., octobre 1957, Tananarive.

20. GRETILLAT (S.). - Maıntien du Genre Bothriophoron Stiles ef Goldberger, 1910, et valeur de l'espèce Paramphistomum bothirophoron (Braun, 1912) Fischoeder, 1901 (Trematoda : Paramphistomidae) parasite du reticulum du zébu malgache. Ann. Paras. hum. comp., 1958, 33, 240-253. 
21. GRETILLAT (S.). - Note préliminaire sur la Gastrothylose des jeunes zébus à Madagascar. Rev. Elev. Med. Vet. Pays trop., 1957, 10, 221-230.

22. GRETILLAT (S.). - Contribution à la connaissance des hôtes intermédiaires et à l'étude du Cycle évolutif de Paramphistomum cervi (Schrank, 1790) à Madagascar. Rev. Elev. Med. vet. Pays trop., 1958, 11, 427-438.

23. GRETILLAT (S.). - Infection à Amphistomata à Madagascar. Hôtes intermédiaires ef épidemiologie de ces parasites à Madagascar. Comm. présentée au Symposium sur les Helminthioses des animaux domestiques du 23 au 25 juillet 1959 à Muguga-Nairobi-Kenya. Publication no 49 CSA/CCTA.

24. JOYEUX (C.), BAER et GAUD. - Recherche sur les Cestodes d'Indochine ef sur quelques Diphyllobothrium (Bothriocephales). Bull. Soc. Path. exot., 1950, 43, 482-489.

25. POISSON (H.). - Prodromes d'éfudes de Parasitologie Malgache in Etudes du Laboratoire de Recherches de Service Vétérinaire de Tananarive, 1927, 1, 11-18.
26. POISSON (H.). - Présentation de Photogra phies d'Acuaria spiralis. Bull. Soc. Sci. Med. Madagascar, 1931, p. 50.

27. POISSON (H.). - Sur la présence d'Amœbotœnia sphenoïdes à Madagascar. Rec. Méd. Vet. exot., 1934, p. 150.

28. POISSON (H.) ef BUCK (G.). - Note à propos de Setaria labiato-papillosa. Rev. Path. exot, 1936, 933-934.

29. POISSON $\left(\mathrm{H}_{1}\right)$ et BUCK (G.). - Une épidémie de Bunostomose sur des Veaux zébus. Comm. Soc. Scl. med. Madagoscor. Déc. 1937.

30. QUENTIN (J. C.). - Description de Cotugnia daynesi, n. sp., Cestode parasite de la poule domestique d̀ Madagascar. Bull. Soc. Path. exot., 1963, 56, 243-251.

31. TISSIE. - Rapport sur les discussions de la 5 Conférence Vétérinaire panafricaine de Nairobi, 1924, p. 92.

Rapports annuels du Service Vétérinaire (Laboratoire) Tananarive et Rapports Annuels du Laboratoire Central de l'Elevage Tananarive (de 1956 d̀ 1963) (Rapports Labelva). 